Monográfico «El impacto de las redes sociales en la enseñanza y el aprendizaje» ARTÍ́CULO

\title{
Utilización de redes sociales
} para la práctica pedagógica en la enseñanza superior impartida en Francia: perspectivas del educador y del estudiante*

\section{Dra. Rachel Panckhurst}

rachel.panckhurst@univ-montp3.fr

Universidad de Montpellier 3

\section{Debra Marsh}

debramarsh@gmail.com

iConnect

\section{Cita recomendada}

PANCKHURST, Rachel; MARSH, Debra (2011). «Utilización de redes sociales para la práctica pedagógica en la enseñanza superior impartida en Francia: perspectivas del educador y del estudiante». En: «El impacto de las redes sociales en la enseñanza y el aprendizaje» [monográfico en línea]. Revista de Universidad y Sociedad del Conocimiento (RUSC). Vol. 8, n.o 1, págs. 233-252. UOC. [Fecha de consulta: $\mathrm{dd} / \mathrm{mm} / \mathrm{aa}]$

<http://rusc.uoc.edu/ojs/index.php/rusc/article/view/v8n1-panckhurst-marsh/v8n1-panckhurstmarsh>

ISSN 1698-580X

* En el marco del equipo de investigación Praxiling UMR 5267 CNRS Universidad Paul-Valéry Montpellier 3 


\title{
Resumen
}

Con el objetivo de estudiar y evaluar las ventajas y desventajas relativas de la aplicación de redes sociales en la enseñanza superior, se exploró, en un período de tres años (2007-2010), el uso de estas herramientas en los cursos de posgrado de aprendizaje electrónico impartidos en Francia. Todos los estudiantes que participaron en el estudio vivían fuera del campus y seguían el plan de estudios del máster en Gestión del conocimiento, aprendizaje y aprendizaje electrónico del Departamento de Lingüística de la Universidad Paul-Valéry de Montpellier 3: http://www.univ-montp3.fr/metice/_masterprogaf/.

Las autoras (que también fueron las tutoras del curso) establecieron un entorno de aprendizaje en colaboración y una comunidad de práctica en línea, e invitaron a sus alumnos a utilizar estas herramientas para debatir cuestiones pedagógicas relacionadas con la práctica del aprendizaje electrónico utilizando una red privada de intercambio de aprendizaje electrónico (eLEN, Marsh et al., 2007; Panckhurst et al., 2008a, 2008b, 2009, 2010), mediante la herramienta de red social Ning (http:// wWw.ning.com).

El objetivo del experimento, que se desarrolló durante tres años, fue situar a los estudiantes en el centro del proceso de aprendizaje, permitiéndoles experimentar y reflexionar sobre el aprendizaje colaborativo en línea, y al mismo tiempo participar en proyectos prácticos específicos.

Este artículo describe y analiza el último estudio de caso realizado (2009-2010) y lo compara con los cuatro estudios de caso anteriores. En el estudio de caso 5, que se llevó a cabo de octubre de 2009 a marzo de 2010, se produjo un cambio significativo en el diseño del aprendizaje (identificado en Panckhurst et al., 2009). Se prestó mayor atención al aprendizaje social (Weller, 2008), centrando el diseño pedagógico en proyectos asignados específicos y no en discusiones individuales. La finalidad de las autoras era comprobar si este cambio pondría en peligro la diversidad, la autonomía, la apertura y la interacción, que son palabras clave asociadas al uso de redes (Downes, 2008). Este artículo aborda estas consideraciones y analiza cómo se ha transformado el papel del tutor para pasar del control a la influencia sutil o a la configuración inicial (Siemens, 2010). El papel del estudiante también ha experimentado un cambio de perspectiva, ya que la pedagogía centrada en el profesor ha sido reemplazada por la gestión de un grupo de iguales, la puesta en común, la autonomía y la responsabilidad de cada alumno.

\section{Palabras clave}

aprendizaje electrónico, redes sociales, redes pedagógicas/educativas, comunidades de práctica, objetos de aprendizaje social, aprendizaje en colaboración / autónomo

\section{Using Social Networks for Pedagogical Practice in French Higher Education: Educator and Learner Perspectives}

\author{
Abstract \\ Social networks were used within graduate elearning French university courses over a three-year period \\ (2007-2010) in order to explore and evaluate the relative advantages and challenges for the usage of
}


such tools in higher education. All students involved were off-campus, and they were following curricula as part of a master's degree in Knowledge Management, Learning and elearning within the Linguistics Department at Paul-Valéry Montpellier 3 University: http://www.univ-montp3.fr/metice/_masterprogaf/.

A collaborative learning environment and an online community of practice were established by the authors (who were also the tutors of the course), and students were invited to use them to discuss pedagogical issues relating to eLearning practice via a private eLearning Exchange Network (eLEN, Marsh \& Panckhurst, 2007, Panckhurst \& Marsh, 2008a, 2008b, 2009, 2010), using the social networking tool Ning (http://www.ning.com).

The aim of the three-year experiment was to place students at the core of the learning process and enable them to experience and reflect upon collaborative online learning while engaged in specific practical projects.

This article describes and analyses the latest case study (2009-2010) and compares it with the previous four case studies. In case study 5, which ran from October 2009 to March 2010, there was significant change in the learning design (identified in Panckhurst \& Marsh, 2009). A social learning object focus (Weller, 2008) was adopted and the pedagogical design was centred on specific imposed projects rather than on individual-led discussions. The authors were keen to check whether this shift would compromise diversity, autonomy, openness and interaction, which are keywords associated with network usage (Downes, 2008). This article addresses these considerations and discusses how tutor/educator roles are currently shifting from control to subtle influence and/or initial shaping (Siemens, 2010). Student/learner roles have also changed perspective, as teacher-centric pedagogy is replaced by peer-group management, collaborative sharing, autonomy and student responsibility.

\section{Keywords}

elearning, social networking, pedagogical/educational networks, communities of practice, social learning objects, collaborative/autonomous learning 


\section{Introducción ${ }^{1}$}

Entre mayo de 2007 y marzo de 2010 se llevaron a cabo cinco estudios piloto utilizando redes sociales en línea con estudiantes de segundo año de máster de la Universidad Paul-Valéry Montpellier 3. Los estudiantes seguían el plan de estudios del máster del Departamento de Lingüística de la universidad, titulado «Gestión del conocimiento, aprendizaje y aprendizaje electrónico» (http://www.univmontp3.fr/metice/_masterprogaf/). La investigación se realizó con estudiantes de fuera del campus, salvo una semana intensiva que transcurrió obligatoriamente en el campus a mediados de curso. El principio subyacente era explorar y evaluar el uso, las ventajas y las dificultades de las herramientas que ofrecen las redes sociales en la enseñanza superior.

Las autoras (que también fueron las tutoras del curso) establecieron un entorno de aprendizaje en colaboración y una comunidad de práctica en línea, e invitaron a sus alumnos a utilizar estas herramientas para debatir cuestiones pedagógicas relacionadas con la práctica del aprendizaje electrónico utilizando una red privada de intercambio de aprendizaje electrónico (eLEN, Marsh et al., 2007; Panckhurst et al., 2008a, 2008b, 2009, 2010), mediante la herramienta de red social Ning² (http:// www.ning.com).

El objetivo del experimento, que se desarrolló durante tres años, fue situar a los estudiantes en el centro del proceso de aprendizaje, permitiéndoles experimentar y reflexionar sobre el aprendizaje colaborativo en línea, y al mismo tiempo participar en proyectos prácticos específicos.

En este artículo, las autoras reflexionan sobre la experiencia que han compartido durante tres años, comparando los distintos estudios piloto realizados, que pueden dividirse en dos fases significativas (eLEN1: estudios de caso 1 a 4; eLEN2: estudio de caso 5). También analizan las razones por las que los profesores universitarios escogen (o podrían escoger en el futuro) utilizar redes sociales para la práctica pedagógica en la enseñanza superior. Otras cuestiones que se debaten son las siguientes: ¿las redes sociales difieren de otras herramientas utilizadas actualmente, como foros, chats, etc??; en caso afirmativo, ¿en qué sentido?; si se adoptan redes sociales en la enseñanza superior, ¿cómo deberían establecerse y utilizarse de modo efectivo?; cuando se utilizan, ¿cambian los papeles del profesor, educador, tutor, estudiante y alumno?; en caso afirmativo, ¿cómo lo hacen?

\section{Estudios de caso}

\subsection{Organización (estudios de caso 1 a 4)}

Los cuatro primeros estudios de caso se llevaron a cabo de mayo de 2007 a marzo de 2009. Los foros en línea, en los que los estudiantes discutían cuestiones pedagógicas relacionadas con la práctica del

\footnotetext{
1. Las autoras quieren dar las gracias a sus alumnos de máster, quienes, a lo largo de los últimos tres años, han constituido una fuente enriquecedora de investigación pedagógica. Los estudiantes han enseñado muchas cosas a sus tutoras durante este periodo.

2. Se eligió Ning por dos motivos: en primer lugar, en ese momento no estaba tan extendida como otras herramientas y, en segundo lugar, la interfaz ya estaba traducida al francés (a diferencia de otras herramientas para redes sociales), factor de gran importancia para nuestros estudiantes.
} 
aprendizaje electrónico, se iniciaron mediante una eLEN privada utilizando Ning. ${ }^{3}$ Se estableció un periodo de dos o tres fases centradas en el estudiante:

- Una fase inicial para que se conociesen entre sí (iniciada y dirigida por las tutoras).

- Una fase de discusión obligatoria, diseñada y dirigida por los alumnos con participación de sus compañeros de grupo.

- Una tercera fase final dirigida por la tutora (nueva en el estudio de caso 4), inspirada en los puntos clave identificados en las discusiones de la fase 2.

\subsection{Organización (estudio de caso 5)}

En el estudio de caso 5 (de octubre de 2009 a marzo de 2010), se produjo un cambio importante en el diseño del aprendizaje (identificado en Panckhurst et al., 2009). Se prestó mayor atención al aprendizaje social (Weller, 2008) para esta eLEN (http://reelgaf2010.ning.com/), aplicando una nueva generación de redes de intercambio de aprendizaje electrónico (eLEN2). El diseño pedagógico se centró en proyectos asignados específicos (véase un ejemplo en el apéndice 2) y no en discusiones dirigidas por individuos.

El estudio de caso 5 se organizó de la forma siguiente: en vez de pedir a los estudiantes que iniciaran y moderaran hilos de discusión individuales, se dividió al grupo de 21 estudiantes en cinco subgrupos de cuatro o cinco miembros cada uno, y a cada uno se le asignaron proyectos específicos (cuyo contenido las autoras esperaban que actuaría como estimulación objetual del aprendizaje social) durante un periodo de dos meses, junto con información de ayuda y enlaces a sitios web. Se estipuló un calendario preciso (apéndice 1) que contenía cinco plazos de entrega por subgrupo y se activaron alertas antes de cada plazo de entrega. Los subgrupos trabajaban en áreas privadas, que sólo eran accesibles a sus miembros, antes de presentar los resultados a la totalidad del grupo. El trabajo de todo el grupo también se inició en fases específicas. Los cinco subgrupos se ajustaron a los plazos de entrega y en alguna ocasión incluso presentaron su trabajo por adelantado, lo que, según la experiencia de las autoras, es algo muy poco frecuente en la enseñanza superior en Francia.

Las tutoras evaluaron el trabajo de cada grupo en marzo de 2010 según cinco criterios:

1. Organización dentro de los subgrupos, herramientas utilizadas, eficiencia de la comunicación interna, observancia de los plazos de entrega.

2. Calidad de la primera síntesis interactiva enviada a la totalidad del grupo.

3. Animación y participación en el foro por parte de sus miembros durante un periodo de dos semanas.

4. Síntesis final: herramientas utilizadas, documentos elaborados.

5. Calidad del contenido final. 


\subsection{Utilización de redes sociales o redes de intercambio de aprendizaje electrónico (eLEN) en la enseñanza superior}

Los análisis de los tres estudios de caso realizados en 2007-2008 sugieren con toda certeza que, más allá de los entornos formales de aprendizaje virtual basados en instituciones (VLE) o los sistemas de gestión de aprendizaje (LMS), las redes sociales pueden resultar beneficiosas para el aprendizaje individual y en colaboración, ya que no sólo proporcionan una mayor sensación de libertad en comparación con las supuestas limitaciones de los VLE y LMS académicos, sino que además animan a los estudiantes a ser más independientes y a asumir una mayor responsabilidad en su propio aprendizaje.

La comunidad de práctica en línea, basada en una herramienta de redes sociales, ha demostrado ser un apoyo importante para los estudiantes que estaban acostumbrados a una pedagogía más tradicional, directiva, centrada en el profesor y guiada (Marsh et al., 2007; Panckhurst et al., 2008a, 2008b, 2009).

Las redes de intercambio de aprendizaje electrónico (eLEN) que hasta el momento habían obtenido resultados positivos incluían los siguientes elementos (véase Panckhurst et al., 2008a, 2009 para conocer los detalles descriptivos):

\section{- Motivación}

- Cohesión de grupo

- Cambio de orientación: de la guía del tutor a la gestión de un grupo de iguales

- Fomento y promoción de la responsabilidad, la independencia y la autonomía del estudiante

- Sentido de pertenencia de los estudiantes

- Mayor libertad a los alumnos por parte del profesorado, que permanece en un segundo plano

El cuarto estudio de caso se estableció a partir de los resultados positivos que se habían obtenido previamente. Sin embargo, desde el comienzo, quedó claro que este grupo no evolucionaría ni se desarrollaría siguiendo las mismas pautas que los que habían participado en los tres estudios de caso anteriores, ya que se había observado un cambio importante en la actitud y la percepción de los estudiantes respecto al valor de las redes sociales y el lugar que estas ocupaban en su aprendizaje. Las autoras/tutoras se preguntaban si las redes sociales estaban simplemente quedando desfasadas del mismo modo que el correo electrónico, los foros, los chats, los blogs, etc. habían quedado aparentemente obsoletos en círculos pedagógicos como consecuencia de los sucesivos cambios tecnológicos. Tras un periodo relativamente breve de dos años, era evidente que la novedad de la interacción en línea, tal como la habían percibido inicialmente los estudiantes, se había convertido en algo trivial y adocenado como resultado del mayor uso privado de herramientas como Facebook ${ }^{4}$ y Twitter $^{5}$. Era evidente que esperaban algo más de una red social utilizada como herramienta de aprendizaje. Así pues, ¿qué debía hacerse?

4. <http://www.facebook.com>

5. <http://twitter.com> 
Cuando las autoras se dieron cuenta de que el valor de novedad quizá se había agotado, era ya demasiado tarde para iniciar una revisión del curso y rediseñar su estructura, de modo que se mantuvo intacto durante el estudio de caso 4. Sin embargo, al final de la sesión y mientras se llevaba a cabo el análisis crítico de investigación, las autoras decidieron que los objetos de aprendizaje social que «facilitan la conversación y por consiguiente la interacción social» (Weller, 2008) debían ser nuevamente definidos en este contexto y puestos en acción mediante una nueva fase de redes de intercambio de aprendizaje electrónico para el siguiente estudio de caso. Este artículo establece las razones para ello y describe cómo las autoras avanzaron hacia la segunda generación de intercambio de aprendizaje electrónico (eLEN2) desarrollando tanto el planteamiento pedagógico como su implantación, lo que a su vez tuvo un gran impacto en el papel y la perspectiva de los educadores y los alumnos.

\subsection{Estudio de caso 5: análisis y resultados}

\subsubsection{Objetos de aprendizaje social impuestos y estructurados}

La estructura no compromete necesariamente la autonomía del estudiante.

Tal como hemos especificado anteriormente, en el estudio de caso 5, las autoras se distanciaron de la moderación del hilo de discusión entre iguales (que había sido la tendencia dominante en los anteriores estudios) para pasar a estructurar proyectos de juego de rol que debían completarse en un margen de tiempo determinado (véanse los apéndices). En un primer momento, a las autoras les preocupaba que este cambio aparentemente radical de planteamiento, con proyectos muy estructurados y plazos estrictos, produjera una pérdida de independencia y autonomía del estudiante. Sin embargo, no fue así. Los resultados del proyecto superaron considerablemente sus expectativas y, en comparación con los anteriores estudios de caso, fueron claramente superiores. Además de asumir la responsabilidad inmediata de su propio aprendizaje, organizando el trabajo en la fase inicial, los subgrupos de estudiantes lograron moderar a la perfección una fase posterior diseñada en torno a la participación de iguales mediante hilos de discusión. También en este caso el contenido pedagógico final y la amplia variedad de herramientas utilizadas tenían una gran calidad.

Los estudiantes explicaron que la imposición de temas comportó un ahorro de tiempo organizacional, y aún así les permitió sentirse independientes y creativos:

\footnotetext{
«En primer lugar, el hecho de que nos impusieran el tema, nos permitió trabajar en cuestiones en las que posiblemente no habríamos pensado, además de representar un ahorro de tiempo importante.

Podíamos empezar inmediatamente a trabajar sin perder dos o tres días escogiendo el tema. Por otro lado, nos permitió tener tiempo para llevar a cabo un trabajo más original e interactivo.»
}

Otro motivo de preocupación era que, por haber establecido plazos y juegos de rol altamente estructurados, los estudiantes recurrieran de nuevo al patrón centrado en el profesor, pidiendo regularmente ayuda y consejo a las tutoras. Pero no fue así, puede que por varias razones, incluidos los mensajes de ánimo de las tutoras y el claro reconocimiento de la confianza entre tutor y estudiante. 
Mensajes de ánimo

«Por lo que respecta a la presencia de las tutoras, al principio no estábamos seguros de si íbamos en la dirección correcta y publicamos mensajes en el foro con resúmenes de nuestras conversaciones. [Un día] estábamos hablando por Skype cuando recibimos un mensaje de ánimo de Debra, que finalizaba diciendo que podíamos ponernos en contacto con vosotras si teníamos algún problema. Era un mensaje breve, pero suficiente para demostrarnos que aunque teníamos autonomía no estábamos solos. Personalmente eso me dio confianza, y creo que a partir de entonces seguimos trabajando con mayor tranquilidad.»

\section{Confianza tutor-estudiante}

«La autonomía casi total que hemos tenido para llevar a cabo las actividades en Ning ha sido un punto a favor. Nos ha enseñado a organizar tareas en grupo, a contrastar nuestras ideas y nuestras dudas sin tener un profesor que nos guiara. Creo que las tutoras han depositado su confianza en nosotros, lo que ha repercutido en nuestro trabajo. Una vez más, esta situación es comparable a lo que puede esperarse en un entorno de trabajo profesional.»

\subsubsection{Introducción: actividades para romper el hielo}

Las actividades para romper el hielo ayudan a cohesionar el grupo e incrementan la confianza del alumnado.

Durante la sesión que tuvo lugar en el campus a lo largo de una semana, los estudiantes indicaron claramente que se sentían apoyados por sus tutoras y tenían confianza en sí mismos para organizar su propio trabajo y para obtener resultados positivos. Sin embargo, ni la confianza ni la cohesión de grupo se consiguen fácilmente. Durante años se ha abogado por el aprendizaje centrado en el estudiante (Coombes et al., 2003), pero la mayor parte de las veces no se lleva a la práctica de forma realista. Con el aprendizaje electrónico es importante lograr la cohesión de grupo mediante actividades que sirvan para romper el hielo y aumenten la confianza del alumnado. La orientación inicial y unos plazos estrictos resultan esenciales para que los estudiantes asuman la responsabilidad de sus logros y de su aprendizaje autónomo.

En el estudio de caso 5, se introdujeron dos tipos de actividades para romper el hielo antes de iniciar los proyectos de juegos de rol. La primera actividad consistía en una serie de cuatro temas de discusión: 1) Darse a conocer (primera discusión informal de introducción, 7 de octubre de 2009 - 12 de octubre de 2009: 31 mensajes intercambiados); 2) Experiencia de aprendizaje electrónico (discusión sobre el contenido del curso, 8 de octubre de 2009 - 13 de octubre de 2009: 44 mensajes intercambiados); 3) Comunidades de práctica: ventajas y desventajas (9 de octubre de 2009 - 2 de noviembre de 2009: 44 mensajes intercambiados); 4) Organización y calendario del semestre (discusión enviada el 10 de octubre de 2009): se pidió a los estudiantes que respondieran en qué momentos no estaban disponibles (aspecto muy importante para la organización de los grupos de trabajo): 27 mensajes enviados hasta el 27 de octubre de 2009. La segunda actividad consistía en un trabajo en grupo durante una semana sobre un tema específico: «El envío de SMS y la enseñanza secundaria y superior» (disponible en http://www.univ-montp3.fr/sl/rachel/M2/SMS.htm). El grupo clase se dividió en dos grandes subgrupos (12 estudiantes en el grupo 1 y 13 en el grupo 2), que tenían solo una semana para leer, 
analizar, compartir, intercambiar y elaborar un resumen sobre el tema, diseñado de forma parecida a los juegos de rol que seguirían posteriormente. Una de las dos tutoras se encargó de orientar a los estudiantes a lo largo de la semana. Las herramientas de comunicación entre iguales incluían el correo electrónico, chats sincrónicos (escritos, orales y a veces en vídeo), foros asincrónicos, redes sociales, teléfonos, etc. Dado que la tutora había especificado que quería tener acceso a cualquier comunicación que se produjera entre los estudiantes fuera de Ning o WebCT, uno de los grupos decidió incluir datos audiovisuales (intercambiados en reuniones de Skype) en el documento final.

Al finalizar la actividad «El envío de SMS y la educación», los estudiantes entregaron un resumen, que debía ser lo más atractivo e interactivo posible. Para la presentación-resumen final, todos los grupos utilizaron distinto software y herramientas complejas, como documentos de procesamiento de texto tradicional, documentos pdf y html, mapas mentales, clústeres de palabras, cuestionarios, hojas de cálculo, diapositivas, documentos audiovisuales (.wmv, .mp4), etc.

\section{Aprender de los compañeros}

Cada subgrupo mostró su producto final al otro subgrupo. Esta comparación entre iguales, además de repetirse en las actividades posteriores de juego de rol, fue muy bien valorada por los estudiantes:

\footnotetext{
«[... ] el hecho de tener acceso al trabajo producido por cada grupo es poco habitual y muy beneficioso; nos aportó una gran cantidad de conocimientos e ideas muy interesantes y útiles para seguir avanzando.»
}

Trabajos en colaboración para romper el hielo y lograr la cohesión de grupo.

A pesar de que los estudiantes admitieron que la semana había sido muy intensa y que era difícil realizar un trabajo en equipo en línea cuando se trataba de un grupo tan numeroso, en retrospectiva estaban convencidos de que había sido una experiencia esencial para romper el hielo y que más adelante les había sido de gran ayuda en los juegos de rol (o en situaciones de la vida real), ya que les había permitido entender con mayor claridad qué se esperaba de ellos en la siguiente fase.

\footnotetext{
«Encontré esta actividad realmente interesante, especialmente desde el punto de vista práctico. Ante todo porque nos permitía trabajar en equipo. Tuvimos que organizarnos y realizar un esfuerzo conjunto para redactar el documento final. Fue un ejercicio de simulación, y es un tipo de experiencia que con toda seguridad tendremos que repetir en el futuro en nuestro entorno laboral.»
}

\section{Autoevaluación, calificaciones y reacciones}

La tutora también pidió a los alumnos que evaluaran el trabajo que habían realizado individualmente y en equipo y situaran su grupo respecto al otro. Algunos estudiantes lo encontraron difícil y no llegaron a entender claramente la intención. Otros, que casi nunca habían realizado autoevaluaciones hasta entonces, estaban intrigados

La tutora calificó los trabajos de los estudiantes según los criterios siguientes (con una nota máxima de cinco puntos): participación asincrónica y sincrónica en los intercambios realizados durante una semana (1,5 puntos); participación en el trabajo en equipo y síntesis final, tanto por la forma 
como por el contenido (2,5 puntos); autoevaluación (1 punto). Luego elaboró un informe de 17 páginas con las calificaciones del estudiante y la evaluación final (puntuada) de su trabajo.

En años anteriores, un informe de 17 páginas (publicado en una de las listas de discusión de Ning) habría suscitado una reacción por parte de los estudiantes (ya fuera positiva o negativa), pero en este caso, curiosamente, no expresaron su opinión sobre el informe, lo que al principio sorprendió a la tutora. En la misma línea, a los estudiantes no pareció interesarles particularmente la calificación que habían obtenido. Se trata de una cuestión fundamental. Cuando las actividades son lo bastante atractivas, la participación y el trabajo en grupo son suficientes. El resto no tiene importancia, o por lo menos pierde importancia y deja de ser el único centro de atención.

«Personalmente, opino que la calificación no es tan importante. Lo que me satisface es que he mejorado en varios aspectos (competencias prácticas y relaciones humanas).»

\subsubsection{Juego de rol: distribución de grupos, apoyo del tutor y observación mutua entre el tutor y el estudiante}

La distribución de grupos no es circunstancial; es conveniente realizar una selección cuidadosa basada en los anteriores intercambios de estudiantes.

Una vez realizadas las actividades iniciales para romper el hielo, los estudiantes están preparados para iniciar las actividades de juego de rol, que permitirán desarrollar la cohesión de grupo, la gestión entre iguales $y$, por consiguiente, la autonomía de los alumnos. De nuevo, estos aspectos no tienen lugar así como así. Las actividades iniciales de discusión, aparentemente informales, y el primer trabajo realizado en grupo (véase sección 2.4.2) son fundamentales para incrementar la confianza entre el tutor y sus alumnos. También son sumamente importantes para que los tutores puedan realizar el seguimiento de los intercambios entre estudiantes y por consiguiente planificar la distribución de grupos al cabo de unas dos semanas. Como se ha especificado anteriormente (en la sección 2.2), se dividió a los estudiantes en grupos de 4 o 5 y no se les permitió escoger con quién iban a trabajar. Las tutoras llevaron a cabo una cuidadosa distribución de estudiantes, según los intercambios que se habían producido en las discusiones anteriores, asegurándose de que los alumnos a los que habían percibido como líderes estuvieran distribuidos equitativamente entre los cinco grupos.

Las tutoras se sitúan en un segundo plano pero aun así los estudiantes se sienten guiados.

Curiosamente, a lo largo de los dos meses que duró la sesión de juego de rol, las tutoras se sentían algo incómodas porque no tenían claro si debían o no intervenir en los intercambios entre estudiantes. Les preocupaba que, si lo hacían, la gestión entre iguales se convirtiera en una comunicación centrada en el profesor, y al mismo tiempo se preguntaban constantemente si este distanciamiento sería beneficioso o perjudicial para los alumnos. Cuando, en diciembre de 2009, tuvo lugar la sesión conjunta en el campus, se les preguntó a los alumnos sobre la actitud de las tutoras, y estuvieron todos de acuerdo en que este había sido uno de los cursos en los que se habían sentido más guiados. En este caso, la impresión de las tutoras había sido completamente distinta; el calendario y los estrictos plazos con tareas específicas de juego de rol, junto con los métodos de alerta (mensajes enviados 24 horas antes como recordatorio durante la sesión de dos meses) y los mensajes de áni- 
mo (véase sección 2.4.1), ayudaron a los estudiantes proporcionándoles plazos de entrega claros y resultados específicos requeridos. Otro factor importante era la idea de que fueran dos las tutoras que dirigieran el curso, ya que cada tutora intervenía a su manera y podían consultarse mutuamente antes de seguir adelante y comunicarse con los estudiantes. Este movimiento de ida y vuelta entre tutoras es también un criterio fundamental para que este tipo de eLEN de búsqueda de autonomía pueda implantarse con éxito.

Supervisión: las tutoras observan a los estudiantes, pero los estudiantes también tienen la oportunidad de observar a las tutoras.

Si bien las tutoras se situaron en un segundo plano y dejaron que los estudiantes siguieran estableciendo su propia organización y colaboración, podían vigilarlos de cerca y comprobar sus intercambios, aunque con escasa capacidad de intervención. Un estudiante, en particular, encontró difícil esta posición:

\footnotetext{
«Aparentemente, las profesoras querían favorecer la autorregulación del estudiante dentro de la red Ning, por lo que casi nunca intervenían, a parte de para poner tareas. Sin embargo, invisible no significa necesariamente ausente, y la mera existencia de esta presencia invisible es suficiente para modificar ligeramente la intención inicial. Como tal, el «ojo controlador», el «inquisidor», está realmente ahí, en la sombra, e induce a un comportamiento no auténtico por parte de los estudiantes.»
}

Sin embargo, cuando se les preguntó por esta cuestión en la sesión de diciembre realizada en el campus, la mayoría de estudiantes afirmó que esta presencia no les perturbaba, y que incluso habían sido capaces de utilizarla en su provecho: en una ocasión, dos estudiantes estaban conversando por chat sincrónico y uno de ellos preguntó si las tutoras realmente habían echado un vistazo a su producción en línea; el otro estudiante respondió: «Sí, ¡una de las tutoras está en línea ahora mismo mirando!». Efectivamente, los tutores pueden observar a los estudiantes, ipero estos también tienen la posibilidad de observarlos a ellos!

\section{Conclusión}

En este artículo las autoras han descrito cómo pueden utilizarse las redes sociales de forma efectiva para la práctica pedagógica en la enseñanza superior en Francia y, posiblemente, también en otros países.

Las redes de intercambio de aprendizaje electrónico (eLEN) pueden utilizarse de forma efectiva si se implementan con esmero, con una planificación específica por parte de los tutores y con la realización de actividades para romper el hielo destinadas a los estudiantes, antes de introducir objetos de aprendizaje social mediante proyectos atractivos. En este sentido, la pedagogía es primordial; se ha de intentar no caer en la trampa de la ilusión tecnológica, en la que la novedad pasa a ocupar una posición central: 
«Aquí no queremos entrar en la "ilusión tecnológica", que ha sido una característica de todas las épocas en las que se han introducido nuevas herramientas pedagógicas (magnetófonos, laboratorios de lenguas, vídeo, ordenadores, etc.). Consideramos que el aspecto esencial de una práctica pedagógica eficiente tiene que ver con el uso, el contenido y el acompañamiento (profesor o tutor) y que las nuevas herramientas o tecnologías deberían facilitar y potenciar estos aspectos.) (Tomé, 2007; Audet, 2010, pág.

78)

El aspecto innovador del trabajo de las tutoras era proporcionar suficientes aportaciones iniciales y una ayuda de base, y luego situarse en un segundo plano. Los estudiantes necesitan seguridad, pero, en este caso, la interacción entre el grupo de iguales resultó ser, en muchas ocasiones, la forma de ayuda más buscada:

«No son los tutores ni el profesorado, sino los que pertenecen a un grupo de iguales los que se implican cada vez más en el proceso de aprendizaje del individuo.» (Ebner et al., 2008; Audet, 2010, pág. 82)

Es preciso asegurarse de que la eLEN incluye suficiente información de cada individuo para que se convierta en una herramienta más personalizada que la mayoría de VLE básicos, por ejemplo, con fotos, breves descripciones individuales, modificación del diseño de la página, etc.:

\footnotetext{
«Una de las mayores ventajas de la red Ning, que fue muy bien utilizada, es la posibilidad de incorporar una foto en el perfil de cada persona. También permite añadir una breve descripción personal, lo que proporciona un toque humano que a menudo no está presente en el aprendizaje electrónico. Todo intercambio va acompañado de la foto del participante, con una interfaz lúdica, modulable, divertida y, por consiguiente, adaptable, lo que contrarresta la naturaleza impersonal de los VLE, como por ejemplo WebCT.»
}

La presentación de la página y las interfaces fácilmente accesibles no son un truco ingenioso; son absolutamente cruciales para fomentar la participación de los estudiantes. Las herramientas deben ser fáciles de usar; la tecnología de perfil bajo y el web abierto poseen grandes ventajas en comparación con los VLE restringidos, que no pueden ser formateados personalmente por los estudiantes.

Utilizar un web abierto no obliga a usar redes sin identificación. En este experimento de tres años de duración sólo se utilizaron eLEN privadas (la totalidad del grupo clase y varios grupos subclase), y no se permitió el acceso a otros colegas de la universidad toda vez que estas se habían establecido específicamente para los estudiantes. Esta implementación estaba basada en el razonamiento pedagógico de que esta red social funcionaba entre un grupo claramente identificado (dos tutoras y los miembros de la clase) y que la interacción y el intercambio se realizaban a partir del conocimiento exacto de quién era quién y de qué papel tenía cada uno dentro de la red. Los colegas que pidieron acceso al grupo no podían ser participantes, sino meros observadores interesados en saber cómo funcionaba, y creemos que esto va contra del mismo fundamento conceptual de una red social. Inicialmente, esto fue un motivo de controversia con nuestros colegas, que en general están acostumbrados a descubrir las innovaciones pedagógicas de otros profesores y están dispuestos a aprender de ellas. 
Sin embargo, al cabo de un año, cuando los estudiantes ya habían dado una respuesta claramente positiva a las organizadoras del curso, la confianza se había depositado e identificado suficientemente para que los demás profesores cambiaran de opinión respecto a la posición del observador. La idea de las tutoras era que al principio debía establecerse un contrato pedagógico que sería usado durante todo el proceso; la confianza y el respeto bidireccionales conducen a un aprendizaje exitoso y ameno.

Una vez se han conseguido la confianza y el respeto, puede establecerse el trabajo en colaboración. El trabajo en equipo sin la intervención continua del tutor es importante. Por ejemplo, en el estudio de caso 5, aunque las tutoras asignaron las mejores calificaciones, las notas pasaron a ser secundarias para los estudiantes, que tenían la impresión de que ya habían aprendido muchas cosas a partir de las actividades en equipo del grupo, un aspecto que se basta por sí mismo. Estos resultados no se habrían podido obtener si las tutoras hubiesen permanecido en un primer plano.

Las tutoras plantean que aunque las herramientas sean de última generación, el diseño pedagógico debe ser sostenible. Los objetos de aprendizaje social (Weller, 2008) tienen que ser atractivos y, si lo son, los estudiantes pueden aportar y crear fácilmente su propio contenido con una orientación inicial del profesor.

Esperar que puedan utilizarse redes sociales en la enseñanza como mera respuesta a una tendencia no es una buena idea. Los estudiantes sólo adoptan una herramienta si perciben que es útil y valiosa para la tarea que están llevando a cabo (Vaufrey, 2009).

El uso pedagógico de redes ha supuesto una transformación de los papeles del educador y del estudiante. Actualmente, el control centrado en el tutor implica organizarse inicialmente y luego situarse en un segundo plano. Al implementar objetos sociales de aprendizaje, la influencia y la forma (Siemens, 2010) ${ }^{6}$ parecen transformarse en una verdadera gestión de grupos de iguales, y los estudiantes parecen sentirse bastante satisfechos de asumir la responsabilidad de su aprendizaje y compartir sus experiencias de forma autónoma, así como de lograr excelentes resultados sin la influencia o el condicionamiento de sus tutores:

«Los profesores ya no asumen toda la responsabilidad por el contenido y aceptan compartir su autoridad; al mismo tiempo, este cambio de paradigma exige que los estudiantes sean más activos y tengan mayor confianza en sus capacidades.» (Audet, 2010, pág. 77)

Así, pues, la organización y la estructura inicial por parte de las tutoras, junto con unos plazos claros, aumentaron la confianza entre tutor y estudiante y, a su vez, lograron una mayor interacción, puesta en común, colaboración, gestión de grupos de iguales y, aún más importante, incrementaron la autonomía y la responsabilidad del alumno.

Ahora los estudiantes ocupan el centro del escenario y deberían quedarse ahí; los profesores están entre bastidores, por si se precisa su ayuda, pero sin intervenir innecesariamente. La confianza

\footnotetext{
6. «Dado que la coherencia y la lucidez son claves para entender nuestro mundo, ¿de qué modo los educadores enseñan en las redes? Para los educadores, el control es sustituido por la influencia. Actualmente, en vez de controlar un aula, el profesor influencia una red o le da forma.» (Siemens, 2010, artículo en blog)
} 
inicial (mediante actividades para romper el hielo) debería indicar claramente a los estudiantes que sus tutores tienen la seguridad de que sus alumnos son capaces de encontrar su propio camino de aprendizaje y no están obligados a seguir el que les han marcado.

\section{Bibliografía}

ANDERSON T. (2009). «Social Networking in Education» [documento en línea]. Capítulo del manual STRIDE. Indira Gandhi National Open University (IGNOU)

<http://terrya.edublogs.org/2009/04/28/social-networking-chapter/>

AUDET, L. (2010). «Wikis, Blogues et Web 2.0 : Opportunités et impacts pour la formation à distance» [documento en línea].

<http://www.educnet.education.fr/dossier/travail-apprentissage-collaboratifs/bibliographie>

BATIER, C.; LEBRUN, M. (2009, enero). «Quels rôles ont les réseaux sociaux dans l'enseignement ?» [en línea]. Les causeries Spiral. <http://apprendre2point0.ning.com/profiles/blogs/quels-roles-ont-les-reseaux>

CARRE, P. (2005). L'Apprenance, vers un nouveau rapport au savoir. París: Dunod.

CONOLE, G.; CULVER, J.; WILLIAMS, P. [et al.] (2008). «Cloudworks: Social networking for learning design» [ponencia en línea]. En: Actas de ascilite (2008: Melbourne). <http://www.ascilite.org.au/conferences/melbourne08/procs/conole.pdf>

COOMBES, S.; PENNY, R.; SMITH, I. (2003). «Improving personal learning through critical thinking scaffolds» [ponencia en línea]. En: Congreso Anual de la Asociación de Investigación Británica (11-13 de septiembre de 2003: Edimburgo). Universidad Heriot-Watt.

<http://www.leeds.ac.uk/educol/documents/00003202.htm>

DOWNES, S. (2008). «Web 2.0, e-Learning 2.0 and the New Learning» [ponencia en línea]. En: Congreso de Tecnologías del Aprendizaje (enero de 2008: Londres).

$<$ http://www.downes.ca/cgi-bin/page.cgi?presentation=173>

EBNER, M.; SCHIEFNER, M. (2008). «Will e-learning die?» [documento en línea]. En: A. R. LIPSHITZ, S. P. PARSONS (editores). E-Learning: 21st Century Issues and Challenges. Nova Publishers. Págs. 69-82.

<http://lamp.tu-graz.ac.at/ i203/ebner/publication/08_nova.pdf>

ENGESTRÖM, J. (2005, 13 de abril). «Why some social network services work and others don't - Or: the case for object-centered sociality» [entrada de blog]. En: Zengestrom.

<http://www.zengestrom.com/blog/2005/04/why_some_social.html>

MARSH, D.; PANCKHURST, R. (2007). «eLEN - eLearning Exchange Networks: reaching out to effective bilingual and multicultural University collaboration» [ponencia en línea]. En: Actas del EADTU (noviembre de 2007: Lisboa). 11 páginas.

<http://www.eadtu.nl/conference-2007/>

PANCKHURST, R.; MARSH, D. (2009). «eLEN2 - 2nd generation elearning Exchange Networks» [ponencia en línea]. En: Actas de Online Educa (diciembre de 2009: Berlín). Págs. 245-248.

$<$ http://www.online-educa.com> 
PANCKHURST, R.; MARSH, D. (2010), «Is social networking moving pedagogical barriers? Using electronic communication for putting social learning objects into action», La communication électronique en situations mono et plurilingues, Coloquio internacional, Universidad de Havre: 9-10 de diciembre de 2010.

<http://www.colloquelehavre2010.org/?p=363>

PANCKHURST, R., MARSHD. (2008b). «REEL: réseaux d'échanges pédagogiques en elearning. Améliorer la qualité de l'apprentissage en favorisant l'autonomie des apprenants» [ponencia en línea]. En: C. CHARNET;C.GHERSI;J.-L.MONINO (coords.) (2008).Actasdel25. CongresoAIPU,Ledéfidelaqualitédans l'enseignement supérieur: vers un changement de paradigme (19-22 de mayo de 2008: Montpellier). $<$ http://www.aipu2008-montpellier.fr>

Actas en línea:

<http://www.aipu2008-montpellier.fr/index.php?dossier_nav=839>

PANCKHURST, R.; MARSH D. (2008a, 4-5 de febrero). «Communities of Practice. Moving from Institutional Platforms to the Open Web as a Platform». París. 6 págs.

$<$ http://www.ilearnforum.eu/>

$<$ http://www.eife-l.org/publications/proceedings/ilf08/contributions/designing-estrategies-forlearning-organisations/panckhurst_marsh.pdf/view>

PAQUET, S. (fecha no especificada). «Impacts sociaux et organisationnels du web social» [curso en línea]. En: INF6107: Le Web Social. Télé-université. <http://benhur.teluq.uquebec.ca/SPIP/inf6107/spip.php?article=79\&rubrique=14>

REFAD [informe en línea]. 99 páginas.

<http://refad.ca/nouveau/Wikis_blogues_et_web_2_0.pdf>

SIEMENS, G. (2010). «Teaching in Social and Technological Networks» [entrada de blog]. Connectivism. $<$ http://www.connectivism.ca/?p=220>

TOMÉ, M. (2007). «Applications pédagogiques des weblogs dans le campus virtuel FLE - Universidad de León» [artículo en línea]. Estudios Humanísticos - Filología. N. 2, págs. 341-373. <http://flenet.rediris.es/tourdetoile/Tome_BlogsCampusEH07.html>

VAUFREY, C. (2009). «Les étudiants créent des sites web pour présenter leurs travaux» [documento en línea]. En: Thot cursus. <http://www.cursus.edu/?module=document\&uid=70684>

WELLER, M. (2008a, 1 de julio). «Social objects in education» [entrada de blog]. The Ed Techie. <http://nogoodreason.typepad.co.uk/no_good_reason/2008/01/whats-a-social.html> 
Apéndice 1: calendario de tareas

\begin{tabular}{|c|c|c|c|c|c|}
\hline Equipo & $\begin{array}{l}\text { Fecha: } \\
\text { composición del } \\
\text { grupo y el tema }\end{array}$ & $\begin{array}{l}\text { Preparación breve } \\
\text { (2 semanas) }\end{array}$ & $\begin{array}{l}\text { Discusión } \\
\text { (2 semanas) }\end{array}$ & $\begin{array}{l}\text { Redacción breve } \\
\text { del documento } \\
\text { sintético } \\
\text { (8 días) }\end{array}$ & $\begin{array}{c}\text { Fecha de entrega } \\
\text { final }\end{array}$ \\
\hline 1 & 20 oct & 21 oct -3 nov & 4 nov - 17 nov & 18 nov - 26 nov & 27 nov \\
\hline 2 & 27 oct & 28 oct -10 nov & 11 nov - 24 nov & 25 nov -3 dic & $04 \mathrm{dic}$ \\
\hline 3 & 03 nov & 4 nov - 17 nov & 18 nov - 1 dic & 2 dic - 10 dic & $11 \mathrm{dic}$ \\
\hline 4 & 10 nov & 11 nov - 24 nov & 25 nov - 8 dic & $9 \mathrm{dic}-17 \mathrm{dic}$ & $18 \mathrm{dic}$ \\
\hline 5 & 17 nov & 18 nov - 1 dic & $2 \mathrm{dic}-15 \mathrm{dic}$ & 16 dic - 7 ene & 08 ene \\
\hline
\end{tabular}

Apéndice 2: Ejemplo de juego de rol en redes sociales asignado al equipo 2

\section{Contexto}

Como resultado de los avances que se han producido recientemente en las tecnologías de aprendizaje, se dispone de una gran variedad de herramientas (blogs, wikis, podcasts, redes sociales, microblogs, etc.), que pueden aportar cambios importantes en las experiencias de aprendizaje de los estudiantes.

Las primeras redes sociales en internet aparecieron en 1995-1996. Algunas personas utilizan términos ligeramente distintos -redes sociales, webs sociales, etc.- con el objetivo de diferenciar entre las redes sociales en internet y el concepto sociológico de redes sociales, que ha existido desde hace mucho tiempo. El interés que suscitan estas redes entre el público es algo más reciente, ya que se inició hacia el año 2000.

«Las redes sociales son un conjunto de tecnologías web 2.0 que permiten crear comunidades de práctica en las que participan las personas que comparten ciertos intereses, aficiones y actividades.» (Panckhurst et al., presentación AIPU, 2008)

Desde hace unos años, el sector de la enseñanza superior también se ha interesado por estas herramientas, porque encajan de forma natural dentro del contexto del web 2.0 o web semántico, y permiten a los participantes desempeñar un papel activo y no simplemente actuar como visitantes de páginas estáticas. Algunos ejemplos de estas herramientas son Facebook (fundada en 2004), MySpace (fundada en 2003), Ning (fundada en 2005) y Elgg (fundada en 2004).

Las plataformas en línea de código abierto para la creación de redes sociales o redes de intercambio de aprendizaje tienen las siguientes características:

- Permiten a los usuarios evitar las limitaciones que supone el uso de una plataforma institucional escogiendo el web abierto. 
- Proporcionan un conjunto de herramientas que están, o pueden estar, directamente integradas (vídeos, chats, foros de discusión, blogs, RSS, fotos, aplicaciones externas, perfiles, etc.).

- Ofrecen numerosas facilidades de uso.

- Utilizan interfaces gráficas sofisticadas e intuitivas.

El último de estos puntos es importante:

«Con el auge del web 2.0, hemos constatado un creciente interés por las herramientas que ofrecen una mayor facilidad de uso y contienen funciones mínimas, en vez de por aquellas que sirven para que todo el mundo pueda hacer de todo. [...] Pero, ¿las herramientas del web 2.0 pueden realmente sustituir a las plataformas de enseñanza a distancia? Según mi opinión, la respuesta es un rotundo sí.» <http://blogs.zdnet.com/web2explorer/?p=337>

Sin embargo, no todo el mundo está convencido de que estas redes sociales/pedagógicas permitan a los estudiantes acceder al aprendizaje «real». ¿Acaso no son tan solo dispositivos tecnológicos complementarios? ¿Tienen realmente algún interés? Una vez la moda tecnológica se centre en otra cosa, ¿no desaparecerán?

Además, han entrado en escena otras herramientas, llamadas microblogs (Twitter, por ejemplo):

«Twitter es un sitio web de microblogging que permite a sus usuarios enviar y leer microentradas de texto de una longitud máxima de 140 caracteres denominadas tweets. El envío de estos mensajes se puede realizar tanto por el sitio web de Twitter como vía SMS desde un teléfono móvil, desde programas de mensajería instantánea [...]»

$<$ http://es.wikipedia.org/wiki/Twitter>

El interés por las redes sociales/pedagógicas y los microblogs está directamente vinculado a la aparición de las tecnologías web 2.0. Los que están a favor de estas herramientas aseguran que pueden ayudar a los estudiantes a asumir una mayor responsabilidad a través de un proceso de aprendizaje semiautónomo y a colaborar mejor con sus compañeros.

Pero, ¿existe alguna prueba de ello? Los que tienen la responsabilidad pedagógica de montar una red de intercambio de aprendizaje, ¿cómo deben acometer tal tarea? ¿Cuáles son sus ventajas y desventajas en comparación con otras herramientas convencionales?

\section{Juego de rol}

El Departamento de Lingüística de la Universidad Paul-Valéry ya ha implantado algunos cursos que utilizan las redes sociales. Consciente de estos avances, la Universidad desea saber con mayor detalle qué ocurre en los países de habla inglesa y otros países europeos para poder: 1) aconsejar mejor a otras universidades que quieren implantar actividades pedagógicas innovadoras y que todavía no han decidido qué herramienta utilizar; 2) cambiar, si procede, las herramientas utilizadas en la universidad (Ning, Facebook), según las conclusiones que se obtengan en el estudio.

La directora del Departamento de Lingüística, Sylvie Gomez-Pescié, se ha puesto en contacto con vuestro grupo de expertos para pediros que presentéis un documento de trabajo que: 
- compare el uso de redes sociales en Francia con el de otros países europeos y los países de habla inglesa;

- sirva de base para la mesa redonda que tendrá lugar en el próximo congreso sobre redes sociales en la enseñanza superior que el Departamento de Lingüística tiene previsto celebrar en la Universidad de Montpellier 3 en 2010.

\section{Objetivo}

Vuestro objetivo es el siguiente:

- Identificar las herramientas de redes sociales y los microblogs más utilizados en el contexto pedagógico en los países de habla inglesa y en Francia.

- Comparar las ventajas y las desventajas de las diferentes herramientas.

- Intentar sugerir la red o las redes que se adapten mejor a la docencia en Francia.

- Indicar las implicaciones y las consecuencias (actuales y futuras) de utilizar estas redes en la enseñanza superior.

- Preparar una evaluación, en forma de documento de trabajo sintético, y entregarlo al experto que supervise vuestro estudio antes de la medianoche del jueves 4 de diciembre.

Tratad de hacer que vuestra presentación sea lo más atractiva e interactiva posible.

\section{Organización}

El grupo está formado por cinco personas: Déborah, Clémentine, Elsa, Andreea y Alexis.

La participación en el foro de Ning es obligatoria.

La organización del trabajo, así como su redacción, depende de vosotros .

\begin{tabular}{ll}
\hline Fechas & Actividad \\
\hline 28 de octubre - 10 de noviembre & $\begin{array}{l}\text { Trabajar en colaboración } \\
\text { Llevar a cabo la investigación } \\
\text { Preparar un informe } \\
\text { Lugar: grupo privado en Ning }\end{array}$ \\
\hline 11 (0 12) de noviembre & Publicar el informe en Ning: espacio general para todos los miembros del curso \\
\hline 11 (0 12) de noviembre - 24 de noviembre & $\begin{array}{l}\text { Crear un hilo de discusión para el tema en Ning } \\
\text { Lugar: espacio general en Ning (para todos los miembros del curso) }\end{array}$ \\
\hline 25 de noviembre - 3 de diciembre & Redactar el documento final sintético \\
\hline
\end{tabular}

\section{Pistas para ayudaros a iniciar el trabajo:}

Congresos internacionales sobre temas de aprendizaje en línea, a distancia y móviles. Los temas son más amplios que las redes sociales, pero podréis encontrar aspectos relacionados:

$<$ http://www.online-educa.com/>

<http://www.conferencealerts.com/elearning.htm>

$<$ http://www.uwex.edu/disted/conf/> 
$<$ http://www.mlearning-conf.org/cfp.asp <

<http://www.academic-conferences.org/icel/icel2009/icel09-home.htm>

23. ${ }^{\circ}$ Congreso Mundial del ICDE: <http://www.ou.nl/eCache/DEF/80/137.html>

Congreso de redes sociales y enseñanza, Universidad de Montreal (marzo de 2009)

$<$ http://www.matimtl.ca/web20.jsp>

\section{Asociaciones}

$<$ http://www.eadtu.nl/>

$<$ http://www.educause.edu/>

$<$ http://www.eden-online.org/eden.php>

\section{En francés:}

Thot - le monde de la formation à distance

$<$ http://www.cursus.edu/>

Buscar «réseaux sociaux» («redes sociales») en el sitio web de Thot

$<$ http://www.cursus.edu/?module=search\&searchString=r\%C3\%A9seaux+sociaux\&searchType= AND\&searchModule $=$ ALL\&subject $=117>$

«Twitter, Facebook et Skype en faveur de la neutralité du web» («Twitter, Facebook y Skype a favor de la neutralidad del web») (20/10/09)

<http://technaute.cyberpresse.ca/nouvelles/internet/200910/20/01-913045-twitter-facebooket-skype-en-faveur-de-la-neutralite-du-web.php>

<http://veillepedagogique.blog.lemonde.fr/2009/07/08/\%C2\%AB-les-reseaux-sociaux-outilsd\%E2\%80\%99apprentissage-en-devenir-applications-a-la-veille-et-a-|\%E2\%80\%99intelligenceeconomique-en-enseignement-superieur-\%C2\%BB/>

Redes sociales y aprendizaje de lenguas

<http://www.cursus.edu/?module=blogs\&action=getPost\&bid=12\&btype=USER\&uid=19> <http://www.francoisguite.com/2009/03/les-reseaux-sociaux-en-education/>

En inglés:

Comparación de Facebook, Ning y Elgg

<http://c4lpt.co.uk/handbook/comparison.html>

<http://web20teach.blogspot.com/2007/08/twitter-tweets-for-higher-education.html>

<http://chronicle.com/blogPost/A-Professor-s-Tips-for-Using/3643>

<http://www.lexrigby.com/2008/11/17/twitter-in-higher-education/>

$<$ http://www.facultyfocus.com/free-report/twitter-in-higher-education-usage-habits-andtrends-of-todays-college-faculty/> 
<http://www.universitybusiness.com/viewarticle.aspx?articleid=1285>

<http://www.classroom20.com/forum/topics/649749:Topic:147280>

<http://nancywozniak.wordpress.com/2008/07/09/the-use-of-facebook-in-education/>

<http://www.miltonramirez.com/2008/11/can-facebook-be-used-in-education.html>

\section{Sobre las autoras}

Dra. Rachel Panckhurst

rachel.panckhurst@univ-montp3.fr

Universidad de Montpellier 3

Profesora / profesora adjunta

Lingüística

Lingüística computacional

Universidad Paul-Valéry de Montpellier 3

Route de Mende

34199 Montpellier Cedex 5

Francia

Debra Marsh

debramarsh@gmail.com

iConnect

Consultora de aprendizaje electrónico miento 3.0 España de Creative Commons. Puede copiarlos, distribuirlos, comunicarlos públicamente y hacer obras derivadas siempre que reconozca los créditos de las obras (autoría, nombre de la revista, institución editora) de la manera especificada por los autores o por la revista. La licencia completa se puede consultar en http://creativecommons.org/licenses/by/3.0/es/deed.es.

\section{$\bullet$ UOC \\ Universitat Oberta \\ de Catalunya \\ www.uoc.edu}

